\title{
Analysis of road safety incentives applied in ppp road
}

\author{
Análisis de incentivos a la seguridad \\ aplicados en concesiones de carreteras
}

Pérez de Villar Cruz, Pablo ${ }^{1}$

\begin{abstract}
:
Road safety is one of the most important issues in PPP roads. At this respect, to achieve a property regulation it is necessary to introduce objective and explicit incentives in the contracts. Besides, these incentives must be focused at the net social benefit.
\end{abstract}

This paper explains how road safety has been introduced traditionally in PPP road contracts and how it is been doing it nowadays, comparing for three recent concessions of Europe, the bonuses and the social benefit associated to each reduction of accidents in the roads. 
As a result, it can be affirmed that the incentives applied, both traditional and the most ones, are unremarkable for two reasons: because they are much lower than the social benefit derived from them and because they apparently are well below the cost of measures to improve road safety.

Keywords: Road safety, incentives, PPP, bonus, social benefit.

\section{Resumen:}

La seguridad de la infraestructura es uno de los aspectos más relevantes en concesiones de carreteras y exige introducir incentivos explícitos y objetivos en los pliegos contractuales para regularla adecuadamente. Además, estos incentivos deben estar orientados a maximizar el beneficio social neto.

En este artículo se recoge cómo se ha regulado este aspecto tradicionalmente en concesiones y cómo se viene haciendo más recientemente, comparando para tres concesiones europeas puestas en servicio en los últimos años, las bonificaciones con el beneficio social que corresponden a cada nivel de reducción de la accidentalidad en la carretera.

Los resultados arrojan que los incentivos aplicados, tanto antiguamente como los más recientes, son anodinos por dos motivos: porque son muy inferiores al beneficio social derivado de ellos $y$ porque aparentemente son muy inferiores al coste de las actuaciones de mejora de la seguridad vial.

Palabras clave: Seguridad Vial, concesiones, incentivos, bonificaciones, beneficio social. 


\section{La finalidad de los incentivos en concesiones de carreteras}

En un mercado perfecto, la calidad se auto-regula y las empresas prestan el servicio ajustando sus condiciones y precio a la demanda. Si los usuarios están dispuestos a pagar por una mejora de calidad rentable de ofrecer, las empresas tienen incentivos para llevarla a cabo, ya que si la acometen aumentará la producción y si no lo hacen pueden perder clientes.

En concesiones de carreteras, cabría pensar en una gestión de la calidad libremente ejercida por las empresas. Sin embargo, se presentan los siguientes impedimentos:

- Al tratarse de un servicio público, las tarifas están reguladas y fijadas de antemano por lo que, en principio, el concesionario no puede recuperar las mejoras de calidad mediante la imposición de nuevos precios, método habitual en los mercados de otro tipo de bienes.

- Como monopolio natural que es, la demanda presenta una baja elasticidad, por lo que el número de usuarios es poco sensible al aumento de la calidad.

- Dada la naturaleza del servicio prestado, puede que los usuarios no valoren las mejoras o incluso que éstas pasen inadvertidas para ellos, lo que hace más difícil justificar un aumento del cobro por su parte.

De estos impedimentos se deduce que la calidad en concesiones de carreteras no puede regularse de forma convencional; concretamente, no puede hacerse depender de la relación tarifas-demanda, por lo que para obtener la calidad deseada hay que introducir expresamente indicadores e incentivos. 
Por otro lado, no hay que olvidar que el objetivo último de la administración titular y reguladora del servicio no es otro que maximizar el beneficio social neto (P. Samuelson, 1947), lo que le impone tres condiciones esenciales:

- Los aspectos de la calidad que se evalúen deben estar lo más directamente relacionados que sea posible con el beneficio social.

- Los incentivos que se introduzcan deben vincularse a los resultados obtenidos graduándolos en función del beneficio social derivado de ellos.

- La formulación de los incentivos debe estar orientada a obtener la calidad más eficiente desde el punto de vista económico-social.

\section{Incentivos a la seguridad vial en concesiones de}

\section{carreteras en servicio}

En los contratos de concesiones de carreteras, la seguridad vial se considera un requerimiento de calidad en materia de conservación y explotación, por lo que tradicionalmente se ha incluido en los correspondientes pliegos como una exigencia general en la prestación del servicio, sin indicadores cuantitativos ni incentivos específicos. De hecho, hasta la década pasada, en el mejor de los casos, los pliegos concesionales se limitaban a establecer unos estándares mínimos relacionados con el estado de conservación de la carretera que si no se cumplían podían implicar sanciones por parte de la Administración (I. G. Heggie and P. Vickers, 1999). Este tipo de contratos se denominan "MOMS", del acrónimo inglés para la expresión "conservación y operación por estándares mínimos" (A. Schliessler and A. Bull, 1994) y todavía es el que está más presente. 
En España, en la mayor parte de las concesiones vigentes, y que de momento vencen entre el 2017 y el 2050, las exigencias de calidad se rigen por disposiciones de hace más de 40 años en las que éstas quedan recogidas de forma muy ambigua. Este es el caso de concesiones sobre importantes carreteras como, por ejemplo, la AP-7 (corredor del Mediterráneo), la AP-2 (corredor Zaragoza-Barcelona) y la AP-1 (corredor Burgos-Bilbao), en las que sigue siendo de aplicación un Reglamento provisional de explotación aprobado por Orden del Ministro de Obras Públicas el 18 de junio de 1969.

Con respecto a los requerimientos relacionados con la seguridad vial, en este reglamento destacan los artículos que se recogen textualmente a continuación:

“Art $3^{\circ}$. El servicio en las autopistas será prestado en condiciones de absoluta normalidad, ..."

“Art. 12. La concesionaria mantendrá en perfecto estado la autopista y sus instalaciones anexas de las áreas de servicio, dentro de las normales condiciones de pulcritud y cuidados estéticos, ..."

“Art 16. La Sociedad concesionaria vendrá obligada a efectuar todos los trabajos de reparación para el mantenimiento de la autopista en perfectas condiciones de utilización, de forma que (...) se evite todo lo que pueda representar peligro para la circulación."

La comprobación de estas obligaciones corresponde a un inspector designado por la Administración, el cual debe evaluar, a su juicio, el estado de la carretera. Las deficiencias observadas debe incluirlas en un informe y también debe proponer las actuaciones que correspondan.

En este sentido, sólo si el concesionario incurre en "negligencia en la conservación de los elementos integrantes de la autopista", 
"deficiencias en la señalización y balizamiento" o "negligencia en la prestación de los servicios al usuario" la Administración queda facultada para la aplicación de multas por día de incumplimiento que pueden liquidarse a través de la fianza depositada en la adjudicación. En última instancia puede procederse al rescate de la concesión.

Sin embargo, desde que se publicó hace más de 40 años el pliego que establece estos preceptos, ni se han actualizado las cantidades previstas (de unos $300 € /$ dia), ni se han ejecutado apenas sanciones por estos motivos. Es más, la práctica habitual es que las sociedades concesionarias auto-regulen las prestaciones del servicio a través de sus propios controles de calidad con poca intervención de los inspectores.

No obstante, desde finales de la década de los ' 90 , se observa una tendencia a recoger explícitamente indicadores e incentivos de seguridad vial en los pliegos de concesiones. La primera concesión en hacerlo fue la M-45, licitada en 1998 por la Comunidad de Madrid en la modalidad de peaje sombra y, desde entonces, las Comunidades Autónomas han adjudicado más de 30 contratos similares sobre una longitud de carreteras superior a los $1.100 \mathrm{~km}$ (J. M. Vassallo and P. Pérez de Villar Cruz, 2010). Por su parte, el Ministerio de Fomento también comenzó a incluir este tipo de cláusulas en sus licitaciones. De hecho, las últimas concesiones de carreteras estatales sin indicadores e incentivos explícitos de seguridad vial fueron las radiales de acceso a la capital, pero las licitadas a partir de entonces sí las han incluido.

Básicamente, los incentivos que se vienen aplicando recientemente son de dos tipos: relacionados con el plazo del contrato o relacionados con los pagos. Cualquiera de los dos tiene repercusiones económicas sobre el concesionario. De hecho, definido un incentivo de un tipo, sería posible encontrar una alternativa equivalente del 
otro tipo en términos de valor actual neto conjugando las variables tarifa, demanda esperada y plazo de concesión. En cualquier caso, los incentivos relacionados con el plazo se han utilizado con mucha menos profusión que los relacionados con el pago. Ejemplos del primer tipo se encuentran en las concesiones del Ministerio de Fomento del Eje Aeropuerto o la recientemente inaugurada MálgaLas Pedrizas. En ellas, la concesionaria puede optar a uno o dos años adicionales de concesión si los indicadores de accidentalidad están una proporción dada por debajo de la media.

Por su parte, la mayoría de los incentivos relacionados con los pagos se limitan a otorgar penalizaciones o bonificaciones en función de la evolución del índice de peligrosidad, comparando el del año a evaluar con el de años anteriores en la misma carretera. Cabe destacar que las bonificaciones o penalizaciones varían notablemente entre diferentes concesiones, aunque generalmente se aplican bajos porcentajes sobre el pago anual aunque los resultados del indicador de seguridad vial sea significativo.

Por ejemplo, para valores del indicador de seguridad vial del orden del $60 \%$ (que indica que el índice de peligrosidad se ha disminuido un $60 \%$ en la misma carretera con respecto al período de evaluación) la bonificación supone el $4 \%$ del pago base en función del tráfico en el período considerado. La tabla 1 (página siguiente) recoge el incentivo establecido para varias concesiones en Galicia (donde se han otorgado 5 contratos sobre más de $100 \mathrm{~km}$ de carreteras), que puede servir para ilustrar cómo se fijan los incentivos a la seguridad vial en numerosas concesiones en España. 


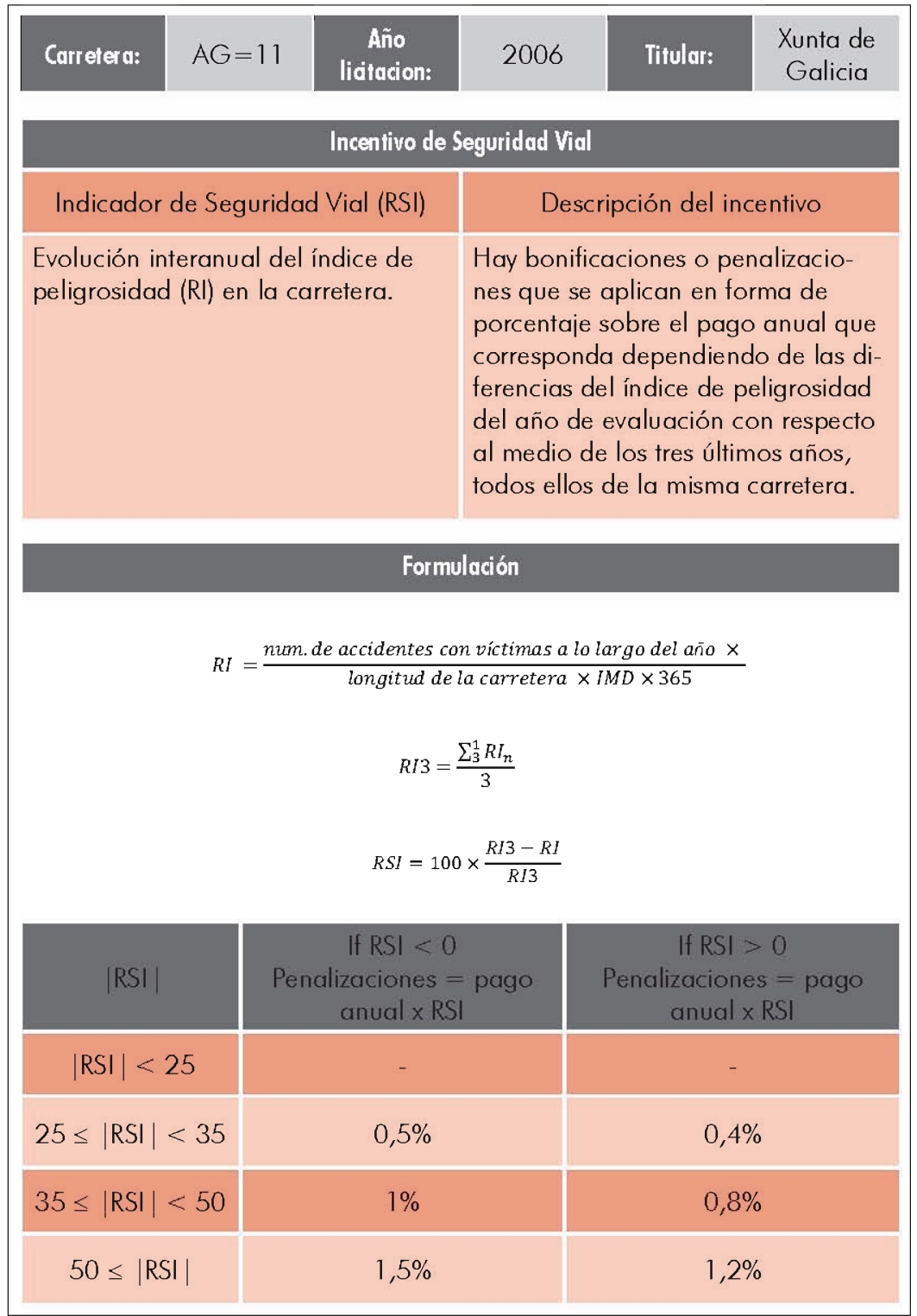

Tabla 1: Ejemplo representativo de indicadores e incentivos a la seguridad vial aplicados en concesiones recientes. 
En el marco del máster universitario en sistemas de ingeniería civil de la Universidad Politécnica de Madrid, se ha llevado a cabo una investigación (Pérez de Villar, P. 2011) para evaluar en qué grado los incentivos más novedosos de seguridad vial introducidos en recientes concesiones del entorno europeo están alineados con el beneficio social.

Con este objetivo, se han escogido tres concesiones con indicadores e incentivos de seguridad vial similares y se han comparado las bonificaciones a otorgar a las concesionarias para cada reducción de accidentalidad en la carretera con los beneficios sociales teóricamente derivados de ellas.

Las concesiones seleccionadas han sido la M-407 (de España), la E18 (de Finlandia) y la IP-4 (de Portugal). En estas concesiones la bonificación anual básicamente consiste en multiplicar el indicador de seguridad vial por el pago anual (o diario). Por su parte, el indicador de seguridad vial es la diferencia del índice de peligrosidad de la carretera en relación con el índice de peligrosidad medio agregado de carreteras similares.

Para comparar la bonificación otorgada con el beneficio social asociados a cada reducción de accidentalidad se han calculado ambas para cada valor del indicador de seguridad vial (RSI), que toma valores de 0 a 100. La bonificación se ha determinado multiplicando cada uno de estos valores por el pago base, que a su vez se ha deducido a partir del canon medio unitario, la intensidad media diaria y la longitud de la concesión. Por su parte, el beneficio social vinculado a cada valor del indicador se ha considerado que es el producto de multiplicar el valor de evitar un accidente con víctimas por la reducción de accidentes teóricamente obtenida.

A partir de cualquier valor del indicador utilizado, es posible obtener los accidentes evitados asociados a dicho valor, siempre que se conozca el índice de peligrosidad de comparación. En la menciona- 
da investigación se han obtenido valores de índices de peligrosidad agregados de las autopistas en el país de cada concesión. Por último, para calcular el valor de evitar un accidente se han introducido los valores propuestos por la Comisión Europea para el cálculo coste-beneficio de inversiones públicas (P. Bickel et al., 2006) en la siguiente expresión:

Por otro lado, como valor de los accidentes evitados se propone emplear el siguiente:

$$
V_{P I A_{s v}}=\frac{\sum_{s r} F A T \cdot V_{F A T}+\sum_{s r} S E I \cdot V_{F A}+\sum_{s r} S L I \cdot V_{S L I}}{\sum_{s r} P I A}
$$

Donde:

- ${ }_{\mathrm{sr}}$ FAT: Número total de víctimas mortales producidas por accidentes de tráfico en carreteras similares a la de la concesión.

- $\mathrm{V}_{\mathrm{FAT}}$ : Valor estadístico oficial de evitar una víctima mortal en carretera en el ámbito geográfico de la concesión.

- S SEI: Número total de heridos graves producidos por accidentes de tráfico en carreteras similares a la de la concesión.

- $\mathrm{V}_{\mathrm{SEI}}$ : Valor estadístico oficial de evitar un herido grave en carretera en el ámbito geográfico de la concesión.

- S SLI: Número total de heridos leves producidos por accidentes de tráfico en carreteras similares a la de la concesión.

- $\mathrm{V}_{\mathrm{SLI}}$ : Valor estadístico de evitar una víctima mortal en carretera en el ámbito geográfico de la concesión.

- ${ }_{\text {sr }}$ PIA: Número total de accidentes con víctimas en carreteras similares a la de la concesión. 
En las figura 1 se recoge, para las 3 concesiones analizadas, la cuantía en euros (representada en el eje de ordenadas) de las bonificaciones y del beneficio social bruto correspondientes a cada valor del indicador de seguridad vial (representada en el eje de abscisas). En ellas puede observarse como los incentivos recogidos en los pliegos están muy por debajo del beneficio social derivado de las mejoras de seguridad vial asociadas a ellos. Además, dichas bonificaciones son aparentemente muy inferiores a los costes de las medidas que sería necesario llevar a cabo para alcanzar los niveles de seguridad correspondientes.

$M \cdot 407$

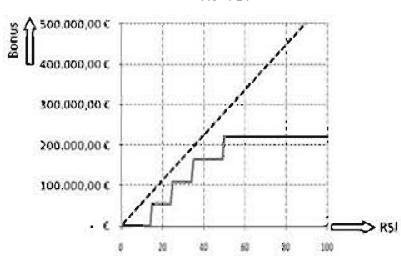

$E 18$

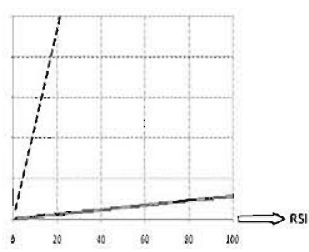

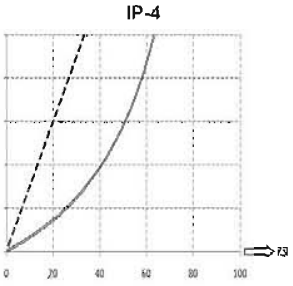

ún los pliegos

- Bonificaclón que correponde a cada RSI

Figura 1: Bonificaciones que se otorgan por la reducción de la accidentalidad en tres concesiones recientemente adjudicadas comparadas con el beneficio social que se deriva de dicha reducción.

\section{Conclusiones}

Según la teoría económica, el fin legítimo y principal de las empresas no es otro que maximizar sus beneficios, mientras que el de la Administración es velar por el interés general. Por este motivo, cuando la gestión de un servicio público se cede al sector privado, deben regularse explícitamente las condiciones del mismo y así conjugar estos diferentes objetivos. 
En la provisión de carreteras, la seguridad viaria es una de las prestaciones más relacionadas con el beneficio económico-social y un aspecto sobre el cual el gestor de la infraestructura tiene una notable capacidad de actuación. Sin embargo, por sus especiales características, su regulación no puede asimilarse al de otros servicios que se prestan en libre mercado. De ello se deduce que en concesiones de carreteras es conveniente introducir incentivos a la seguridad vial que sean explícitos, objetivos y, sobre todo, que estén orientados hacia la maximización del beneficio social neto.

Sin embargo, se ha constatado que tradicionalmente los incentivos a la seguridad vial se han contemplado de forma muy ambigua en concesiones de carreteras y que en muchas de las que vencen a medio y largo plazo no se incluyen indicadores ni incentivos específicos. Por su parte, en las concesiones más recientes del entorno europeo, aunque sí se han introducido bonificaciones específicas, éstas resultan anodinas por su baja cuantía.

A este respecto, sería recomendable que en futuras concesiones de carreteras los incentivos para la mejora de la seguridad vial estuvieran más vinculados y proporcionados al beneficio social derivado realmente de la gestión de la concesionaria para prevenir la accidentalidad. Esto se ha facilitado en España desde la entrada en vigor del Real Decreto 345/2011, de 11 de marzo, sobre gestión de la seguridad de las infraestructuras viarias en la Red de Carreteras del Estado, al obligar a disponer de valores económicos oficiales de evitar accidentes mortales y graves. 


\section{Referencias}

Heggie, I. G. and P. Vickers. 1999. Commercial Management and $F i-$ nancing of Roads. The International Bank for Reconstruction and Development. Washington, D.C.: The World Bank.

Pérez de Villar, P; 2011. Formulación de incentivos óptimos para la mejora de la seguridad vial en concesiones de carreteras. Tesina. Universidad Politécnica de Madrid.

Samuelson, P. (1947). Foundations of Economics Analysis. Cambridge: Mass.

Schliessler, Andreas, and A. Bull. 1994: CAMINOS - Un Nuevo Enfoque para la Gestión y Conservación de Redes Viales, CEPAL, Comisión Económica para América Latina y El Caribe, Naciones Unidas, Santiago de Chile.

Vassallo, J. M. y P. Pérez de Villar Cruz; 2010; Diez años de peaje sombra en España. Revista de obras públicas, 157; 19-30. 